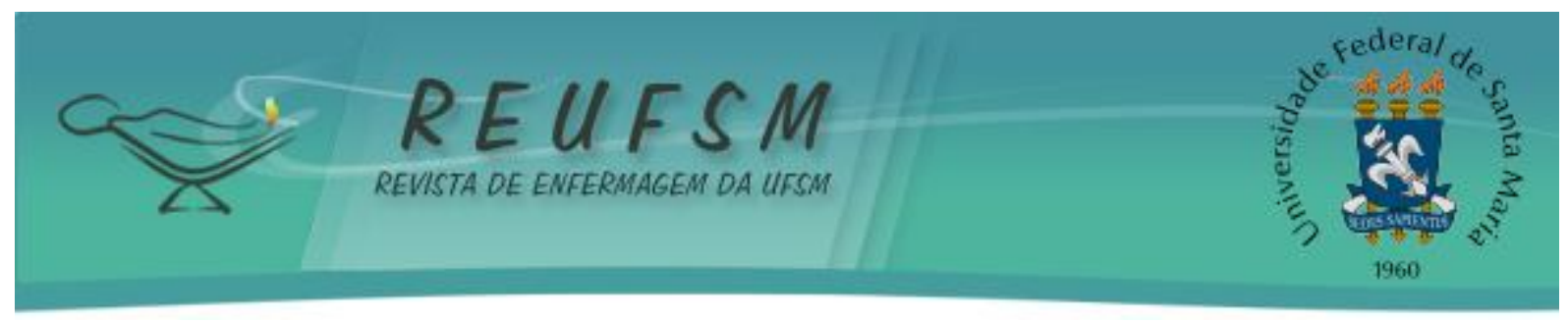

ARTIGO ORIGINAL

\title{
RISCOS ERGONÔMICOS PARA TRABALHADORES DE ENFERMAGEM AO MOVIMENTAR E REMOVER PACIENTES
}

\section{ERGONOMIC RISK FOR NURSING WORKERS IN MOVING AND REMOVING PATIENTS RIESGO ERGONÓMICO PARA TRABAJADORES DE ENFERMERÍA AL TRASLADAR Y MOVER PACIENTES}

Doi: $10.5902 / 2179769215016$

Thiana Sebben Pasa ${ }^{1}$ Tânia Solange Bosi de Souza Magnago ${ }^{2}$ Rosângela Marion da Silva ${ }^{3}$ Anamarta Sbeghen Cervo ${ }^{4}$ Carmem Lúcia Colomé Beck ${ }^{5}$ Natieli Cavalheiro Viero ${ }^{6}$

RESUMO: Objetivo: mapear os riscos ergonômicos para trabalhadores de enfermagem durante procedimentos de movimentação e remoção de pacientes adultos internados em um hospital universitário. Método: estudo transversal, realizado com 123 pacientes adultos internados, entre os meses de fevereiro a abril de 2011. Foram coletados dados referentes à caracterização sociodemográfica e aplicada a Escala de Avaliação do Risco na Movimentação e Transporte de Pacientes. Para organizar os dados foi utilizado o programa Excel $^{\circledR}$ e a análise dos dados foi realizada no PASW Statistic, por meio da análise descritiva. Resultados: obteve-se que 55,3\% dos pacientes apresentaram médio e muito risco ergonômico. Das unidades avaliadas, a Terapia Intensiva e Cardiologia Intensiva foram as que apresentaram maiores riscos ergonômicos, com 83,3\% dos pacientes classificados com muito risco. Conclusão: medidas para reduzir riscos ergonômicos devem contemplar programas de treinamento e capacitação, além de fornecimento de materiais e equipamentos para auxílio na movimentação dos pacientes.

Descritores: Enfermagem; Movimentação e reposicionamento de pacientes; Saúde do trabalhador.

ABSTRACT: Aim: to map the ergonomic risks for nursing workers when handling and moving adult patients in a university hospital. Method: cross-sectional study involving 123 hospitalized adult patients, between the months of February to April 2011. Data regarding sociodemographic characteristics were collected and the Scale of Risk Assessment in Patient Handling was applied. Excel ${ }^{\circledR}$ software was used for data organization and data analysis was developed on PASW Statistics, through descriptive statistics. Results: it was found that $55.3 \%$ of patients had moderate and high ergonomic risk. From the units evaluated, the Intensive Care Unit and the Intensive Cardiology Unit showed the greatest ergonomic risks, with $83.3 \%$ of patients classified as high risk. Conclusion: measures to reduce ergonomic

\footnotetext{
${ }^{1}$ Enfermeira. Mestre em Enfermagem. Universidade Federal de Santa Maria/UFSM - Hospital Universitário de Santa Maria/HUSM/EBSERH. Santa Maria, RS, Brasil. E-mail: thianasp@hotmail.com

${ }^{2}$ Enfermeira. Doutora em Enfermagem. Universidade Federal de Santa Maria/UFSM - Hospital Universitário de Santa Maria/HUSM/EBSERH. Santa Maria, RS, Brasil. E-mail: tmagnago@terra.com.br

${ }^{3}$ Enfermeira. Doutora em Ciências. Universidade Federal de Santa Maria/UFSM. Santa Maria, RS, Brasil. E-mail: cucasma@terra.com.br

${ }^{4}$ Enfermeira. Mestre em Enfermagem. Hospital Universitário de Santa Maria/HUSM/EBSERH. Santa Maria, RS, Brasil. E-mail: anamarta67@yahoo.com.br

${ }^{5}$ Enfermeira. Doutora em Enfermagem. Universidade Federal de Santa Maria/UFSM. Santa Maria, RS, Brasil. Email: carmembeck@gmail.com

6 Enfermeira. Serviço de Atenção Móvel de Urgência. Cachoeira do Sul, RS, Brasil. E-mail: natielivieiro@hotmail.com
} 


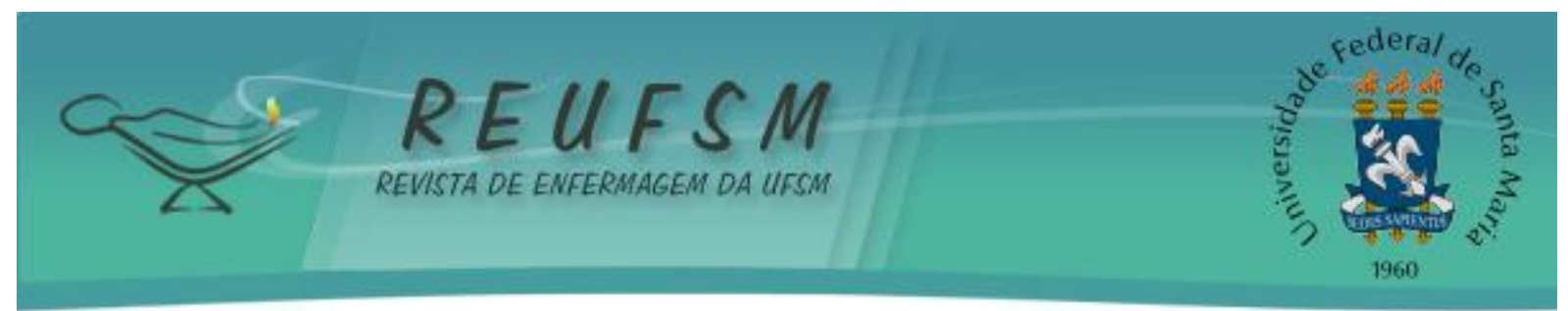

risks should include training and capacity-building programs, as well as supply of materials and equipment to aid in patients handling.

Descriptors: Nursing; Moving and lifting patients; Occupational health.

RESUMEN: Objetivo: mapear los riesgos ergonómicos de los trabajadores de enfermería durante los procedimientos para el transporte y la remoción de pacientes adultos ingresadosen un hospital universitario. Método: estudio transversal, realizado con 123 pacientes adultos ingresados, entre febrero y abril 2011. Fueron recogidas datos sobre características sociodemográficas y aplicada la Escala de Evaluación de Riesgos en el Movimiento y Transporte de Pacientes. Para organizar los datos fue utilizado el programa Excel $\AA$, y el análisis se realizó con el programa PASW Statistics, mediante uso de estadística descriptiva. Resultados: se encontró que 55,3\% de pacientes tenían medio y mucho riesgo ergonómico. De las unidades evaluadas, Cuidados Intensivos y Cardiología Intensiva mostraron mayores riesgos ergonómicos, con $83,3 \%$ de pacientes clasificados como de riesgo muy alto. Conclusión: las medidas para reducir riesgos ergonómicos deben incluir programas de capacitación y suministro de materiales y equipos para ayudar en el movimiento de pacientes.

Descriptores: Enfermería; Movimiento y levantamiento de pacientes; Salud laboral.

\section{INTRODUÇÃO}

A incorporação de novas tecnologias associada ao ritmo intenso de tarefas tem alterado o mundo do trabalho e repercutido na saúde do trabalhador. Na enfermagem, esse ritmo intenso está relacionado ao acúmulo de funções decorrentes da carência de recursos humanos e materiais, o que exige do trabalhador maior energia para desenvolver as suas atividades e tem repercussão na sua saúde física e psíquica. ${ }^{1}$ Neste sentido, as instituições devem promover melhores condições de trabalho, visando melhor qualidade de vida para os trabalhadores.

No Brasil, com o propósito de promover essas questões, o Ministério da Saúde (MS) instituiu a Política do Trabalhador e da Trabalhadora que tem por finalidade definir os princípios, as diretrizes e as estratégias para o desenvolvimento da atenção integral à saúde dessa classe. ${ }^{2}$

Dentre as profissões que merecem atenção no que se refere à saúde do trabalhador está a enfermagem, que exige força física dos trabalhadores durante alguns procedimentos, como na movimentação e remoção de pacientes. O esforço físico necessário para auxiliar na mobilização ou alternância de posição dos pacientes, pode ocasionar lombalgias aos trabalhadores, o que interfere na eficiência da força e capacidade de trabalho da enfermagem. ${ }^{3}$

Estudo apontou que dentre os diversos riscos ocupacionais a que os trabalhadores de enfermagem estão expostos, as queixas relacionadas ao aparelho osteomuscular, representam uma das maiores causas de sofrimento dessa classe trabalhadora, devido ao esforço físico exigido desses profissionais, principalmente durante a movimentação e remoção de pacientes. ${ }^{4}$

Deve-se atentar para os fatores de risco que interferem na saúde durante os processos de movimentação e remoção de pacientes, como condições ergonômicas inadequadas de mobiliários, posto de trabalho e equipamentos utilizados para essas atividades. ${ }^{4}$ Além disso, muitas vezes, a movimentação e remoção de pacientes são realizadas com um quantitativo de profissionais inferior ao desejável e com equipamentos inadequados, o que aumenta o risco de desenvolver problemas osteomusculares. 


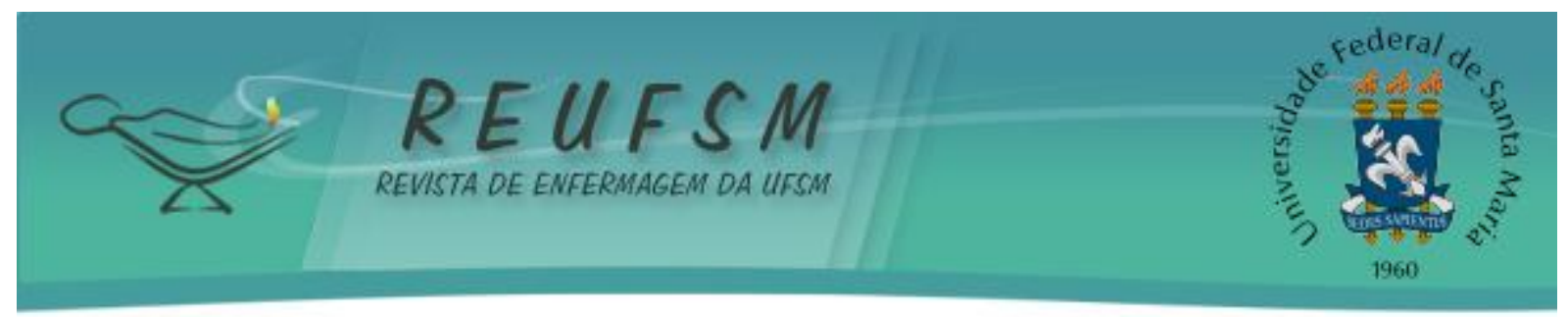

Nesse sentido, percebe-se a importância da ergonomia, ou seja, a necessidade de adequar as condições de trabalho às peculiaridades psicofisiológicas dos trabalhadores, com o intuito de prevenir problemas de saúde a que estes estão expostos. ${ }^{5}$ Torna-se necessário sensibilizá-los em relação à maneira mais adequada de realizar os procedimentos.

Diante dessa problemática, é importante realizar uma abordagem multifatorial, nas quais sejam incorporados os aspectos organizacionais do trabalho, bem como adequações no ambiente físico e características das tarefas, para a prevenção do desenvolvimento dos problemas osteomusculares e diminuição dos riscos ergonômicos. ${ }^{6}$

Esses riscos podem afetar as características psicofisiológicas dos trabalhadores, sendo exemplos o levantamento de peso, transporte e descarga individual de materiais, postura inadequada no trabalho e repetitividade na execução das tarefas, o que causa desconforto e afeta a segurança e saúde dos trabalhadores. Consta na Norma Regulamentadora $\mathrm{n}^{\circ} 17$, de 2007 , do Ministério da Saúde que o peso máximo tolerável para as mulheres e os trabalhadores jovens, quando designados para realizar transporte manual de cargas, deverá ser nitidamente inferior ao dos homens. ${ }^{5}$ Nesse contexto, destaca-se a enfermagem como uma das profissões predominantemente feminina e por ser o maior contingente da força de trabalho nos hospitais, está suscetível a maiores riscos ergonômicos neste cenário.

Pesquisa realizada com trabalhadores de enfermagem de um hospital universitário, evidenciou prevalência de dor ou desconforto músculoesquelético entre os mesmos, sendo a movimentação e remoção dos pacientes um fator contribuinte para a ocorrência dessa situação. ${ }^{7}$

A partir dos resultados do estudo supracitado, este estudo teve por objetivo mapear os riscos ergonômicos para os trabalhadores de enfermagem durante os procedimentos de movimentação/remoção de pacientes adultos internados em um hospital universitário.

\section{MÉTODO}

Trata-se de uma pesquisa transversal, de caráter quantitativo, desenvolvida em um hospital universitário do interior do estado do Rio Grande do Sul, Brasil. O estudo foi realizado em todas as unidades de internação de pacientes adultos: Pronto-Socorro (22 leitos), Clínica Cirúrgica (40 leitos), Unidade de Terapia Intensiva Adulto (UTI) (nove leitos), Unidade de Cardiologia Intensiva (UCI) (quatro leitos), Clínica Médica I (24 leitos; atende as especialidades de hemato-oncologia e cardiologia) e Clínica Médica II (24 leitos; atende pacientes com doenças crônicas, como doença pulmonar obstrutiva crônica, doença de Alzheimer, entre outras), que totalizam 123 leitos. Assim, a população de estudo foi composta pelos 123 pacientes internados nas unidades de estudo, representando uma única avaliação por leito.

O critério de exclusão do estudo era ser menor de 18 anos. A coleta de dados foi realizada por uma das pesquisadoras, no período de fevereiro a abril de 2011, após a aprovação do Comitê de Ética em Pesquisa da Universidade Federal de Santa Maria - UFSM, sob o processo número: 23081.019008/2010-72, em 4 de janeiro de 2011. Foram respeitados os preceitos éticos estabelecidos pela Resolução $n^{\circ}$ 196/96 do Conselho Nacional de Saúde/MS, que dispõe sobre diretrizes e normas regulamentadoras da pesquisa envolvendo seres humanos. 8 Os pacientes foram informados sobre os objetivos da pesquisa e da voluntariedade da participação, sendo a anuência com a pesquisa expressa pela assinatura do Termo de Consentimento Livre e Esclarecido (TCLE), assinado pelo paciente ou em caso de inconsciência ou desorientação deste, era solicitada a assinatura do acompanhante. 


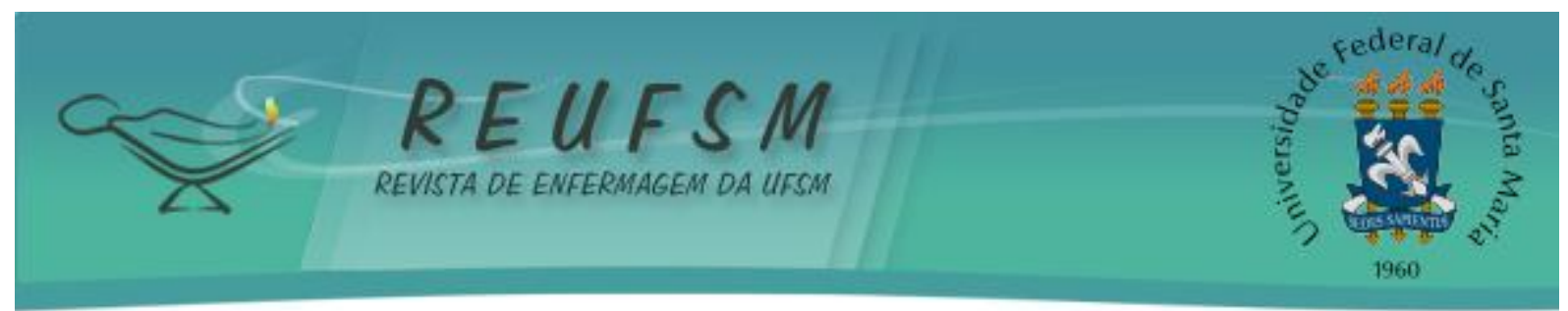

Para a coleta dos dados foi utilizado um formulário contendo dados sociodemográficos (sexo, idade, nível de escolaridade e procedência) e a Escala de Avaliação do Risco na Movimentação e Transferência de Pacientes, validada em pesquisa com pacientes adultos internados em Unidades de Terapia Intensiva (UTI) e de Cardiologia. ${ }^{9-10}$ Essa escala avalia questões relacionadas ao paciente como: peso, altura, nível de consciência e psicomotricidade, mobilidade na cama, transferência da cama/maca para cama/cadeira e vice versa, deambulação, cateteres e equipamentos e ambiente. Cada item varia de 1 a 3 pontos (pouco risco $=1$; médio risco $=2$; muito risco $=3$ ). $\mathrm{A}$ pontuação varia de 8 a 24 pontos. Quanto maior a pontuação, maior é o risco ergonômico que o paciente oferece ao profissional. ${ }^{9}$

A caracterização sociodemográfica, o peso e a altura do paciente foram obtidos por meio de coleta no prontuário. Na ausência dessas informações nos prontuários, recorreu-se à entrevista com o paciente ou acompanhante. A avaliação do risco ergonômico foi realizada junto ao paciente por meio do preenchimento dos itens da escala, utilizando a observação direta e não participante. Nessa atividade, o paciente era observado por aproximadamente 30 minutos em relação ao nível de consciência e psicomotricidade, mobilidade na cama, deambulação, uso de cateteres e equipamentos, bem como quanto à movimentação e remoção (cama/maca para cama/cadeira e vice versa).

Os dados foram organizados em planilha eletrônica do programa Excel $^{\circledR} 2003$ e, após, utilizou-se o programa de análise estatística PASW Statistic ${ }^{\circledR}$ (Predictive Analytics Software, SPSS Inc., Chicago - USA) versão 18.0 para o Windows.

As variáveis contínuas foram expressas como média e desvio padrão, e as variáveis categóricas em frequências absolutas e percentuais. A pontuação do escore de risco ergonômico foi obtida a partir do somatório dos escores: 8 a 12 pontos (pouco risco para o trabalhador durante a movimentação e transferência do paciente), 13 a 18 pontos (médio risco) e 19 a 24 pontos (muito risco). ${ }^{8}$

Para avaliar a associação entre unidade de internação e risco ergonômico, utilizou-se o teste Qui-quadrado, evidenciando associação positiva se $p<0,05$.

\section{RESULTADOS}

Dentre os 123 pacientes analisados, maior percentual era do sexo masculino $(56,9 \%)$ e com idade superior a 56 anos $(52 \%)$. A média de idade foi de 55,3 anos $( \pm 17,8)$ e variou de 19 a 93 anos. No que se refere à escolaridade, 59,3\% cursaram até o ensino fundamental (Tabela 1). Em relação à procedência, 30,1\% eram residentes em Santa Maria e os demais em outras cidades. 


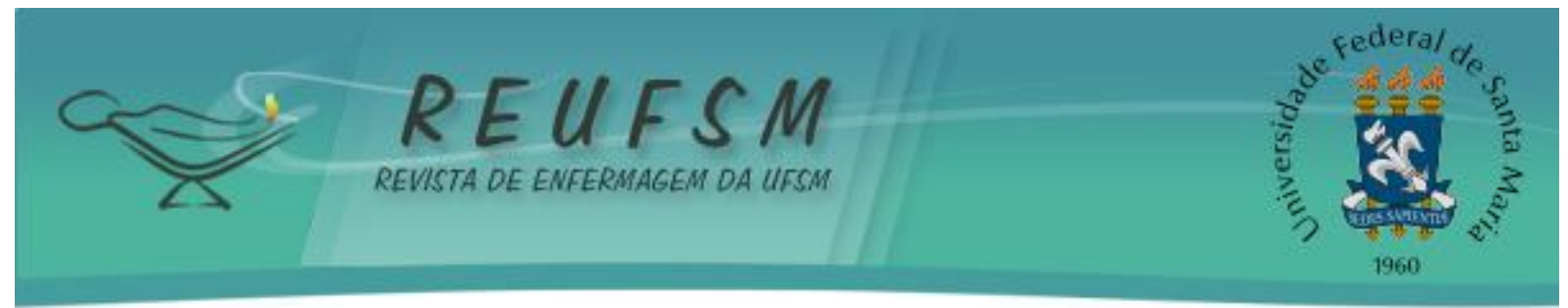

Tabela 1 - Distribuição da frequência dos pacientes, segundo dados sociodemográficos. Santa Maria/RS, 2011. ( $n=123)$

\begin{tabular}{lll}
\hline Características sociodemográficas & N & $\%$ \\
\hline Sexo & 53 & 43,1 \\
Feminino & 70 & 56,9 \\
Masculino & & \\
Faixa etária & 59 & 48,0 \\
Até 55 anos & 64 & 52,0 \\
$\geq 56$ anos & & \\
Escolaridade & 12 & 9,7 \\
Não estudaram & 73 & 59,3 \\
Ensino fundamental (incompleto/completo) & 25 & 20,4 \\
Ensino superior incompleto & 13 & 10,5 \\
Não responderam & & \\
Procedência & 37 & 30,1 \\
Santa Maria & 86 & 69,9 \\
Outras cidades & & \\
\hline
\end{tabular}

A Tabela 2 apresenta os percentuais de acordo com os itens da escala de avaliação dos riscos ergonômicos para os trabalhadores de enfermagem durante os procedimentos de movimentação/remoção de pacientes adultos internados em um hospital universitário, distribuídos em pouco risco, médio risco e muito risco.

Tabela 2 - Frequência das variáveis da escala de acordo com a avaliação do risco ergonômico. Santa Maria/RS, 2011.

\begin{tabular}{lccccccc}
\hline \multirow{2}{*}{ Variável da escala } & \multicolumn{2}{c}{ Pouco risco } & \multicolumn{2}{c}{ Médio risco } & \multicolumn{2}{c}{ Muito risco } \\
\cline { 2 - 7 } & N & $\%$ & N & $\%$ & N & $\%$ \\
\hline Peso & 13 & 10,6 & 53 & 43,1 & 57 & 46,3 \\
Altura & 09 & 7,3 & 94 & 76,4 & 20 & 16,3 \\
Nível de consciência & 93 & 75,6 & 12 & 9,8 & 18 & 14,6 \\
Mobilidade & 77 & 62,6 & 16 & 13,0 & 30 & 24,4 \\
Transferência & 58 & 47,2 & 25 & 20,3 & 40 & 32,5 \\
Deambulação & 56 & 45,5 & 16 & 13,0 & 51 & 41,5 \\
Cateteres & 49 & 39,8 & 45 & 36,6 & 29 & 23,6 \\
Ambiente & 103 & 83,7 & 20 & 16,3 & $\cdots$ & $\ldots$ \\
\hline
\end{tabular}

Em relação aos itens da escala, o peso foi a variável que obteve maior percentual para muito risco ergonômico para trabalhadores de enfermagem $(46,3 \%)$ durante a movimentação e remoção dos pacientes. Relacionado à altura dos pacientes, evidenciou-se que $76,4 \%$ tinham de 1,51 a 1,79 metros, proporcionando médio risco ergonômico aos trabalhadores investigados. 


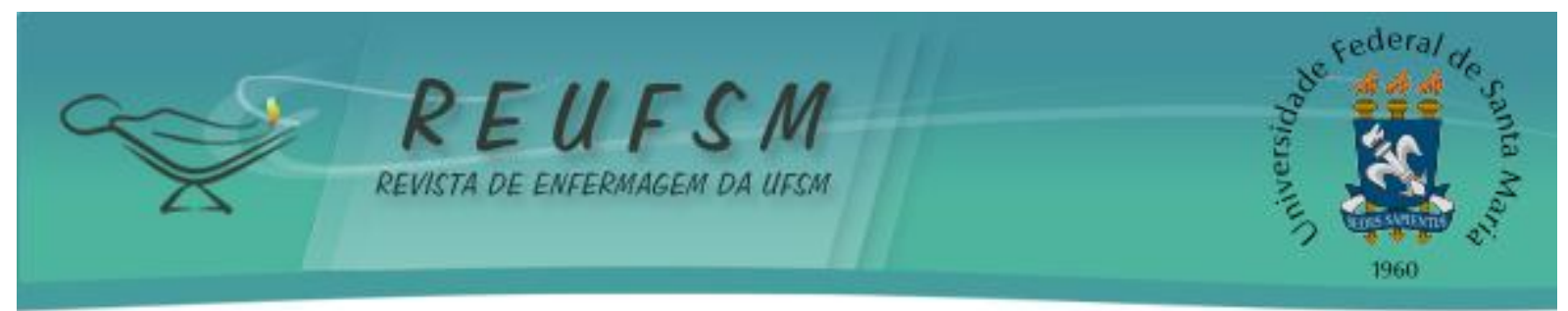

Com relação ao nível de consciência, $75,6 \%$ dos pacientes encontravam-se em estado de alerta, respondendo apropriadamente aos mínimos estímulos. No que tange à mobilidade, $62,6 \%$ dos pacientes conseguiam realizar voluntariamente muitos movimentos no leito, como, por exemplo, movimentar os membros superiores e inferiores, alternar decúbito e sentar-se na cama. Ainda nesse aspecto, em relação ao item da escala "transferência da cama/maca para cama/cadeira e vice-versa", 47,2\% dos pacientes sentavam-se ou alternavam decúbito e se transferiam sem necessitar de auxílio. Relacionado à deambulação, 45,5\% dos pacientes não necessitavam de assistência da enfermagem ou recursos auxiliares.

Quanto ao uso de acessórios e equipamentos, 60,2\% dos pacientes possuíam de dois a mais de cinco acessórios (sondas, drenos, soros, cateteres, bolsas de colostomia e cistostomia) ou equipamentos (monitores, respiradores, bomba de infusão, oxímetros, máquinas de hemodiálise, tração transesquelética e cutânea).

Com relação ao ambiente, $83,7 \%$ dos leitos observados não apresentavam risco durante o processo de movimentação e remoção do paciente. Esse dado pode estar relacionado a alguns elementos do ambiente de trabalho como o espaço físico adequado, piso sem frestas e saliências, camas com altura ajustável, cama/maca, cadeira de rodas e cadeira de banho com travas nas rodas.

Dos 123 pacientes pesquisados, $55(44,7 \%)$ ofereciam pouco risco ergonômico para o trabalhador durante a movimentação e remoção, ou seja, não necessitavam de auxílio, mas requeriam a presença da equipe de enfermagem. Entre os que ofereciam médio risco ergonômico para o trabalhador de enfermagem, 47 pacientes $(38,2 \%)$ exigiam 0 planejamento do enfermeiro em relação aos equipamentos que deveriam ser utilizados e ao número de profissionais que seriam necessários para movimentá-los ou transferi-los. Já 21 pacientes $(17,1 \%)$ apresentavam muito risco ergonômico durante os procedimentos de movimentação e remoção, exigindo rigoroso planejamento, auxílio constante da equipe de enfermagem e de equipamentos mecânicos.

A classificação de risco ergonômico por unidade de internação de pacientes adultos está descrita na Tabela 3.

Tabela 3 - Distribuição das unidades de internação conforme classificação de riscos ergonômicos. Santa Maria/RS, 2011

\begin{tabular}{|c|c|c|c|c|c|c|c|c|c|}
\hline \multirow{3}{*}{ Unidade de internação } & \multicolumn{6}{|c|}{ Classificação de risco ergonômico } & \multirow[t]{3}{*}{ TOTAL } & \multirow[b]{3}{*}{$\mathrm{N}$} & \multirow[b]{3}{*}{$\%$} \\
\hline & \multicolumn{2}{|c|}{ Pouco risco } & \multicolumn{2}{|c|}{ Médio Risco } & \multicolumn{2}{|c|}{ Muito Risco } & & & \\
\hline & N & $\%$ & $N$ & $\%$ & N & $\%$ & & & \\
\hline UTI e UCl* & 01 & 7,7 & 02 & 15,4 & 10 & 76,9 & & 13 & 10,7 \\
\hline Clínica Cirúrgica & 17 & 42,5 & 20 & 50,0 & 03 & 7,5 & & 40 & 32,5 \\
\hline Clínica Médica I & 19 & 79,2 & 04 & 16,7 & 01 & 4,1 & & 24 & 19,5 \\
\hline Clínica Médica II & 10 & 41,7 & 12 & 50,0 & 02 & 8,3 & & 24 & 19,5 \\
\hline Pronto-Socorro & 06 & 27,3 & 11 & 50,0 & 05 & 22,7 & & 22 & 17,8 \\
\hline TOTAL & 53 & 43,1 & 49 & 39,8 & 21 & 17,1 & & 123 & 100 \\
\hline
\end{tabular}

* UTI= Unidade de Terapia Intensiva UCl= Unidade Cardiológica Intensiva

Os dados evidenciaram o dispêndio de esforço físico por parte dos trabalhadores da instituição pesquisada, uma vez que a soma das frequências de pacientes classificados com médio $(39,8 \%)$ e muito risco ergonômico $(17,1 \%)$ totalizou $56,9 \%$. 


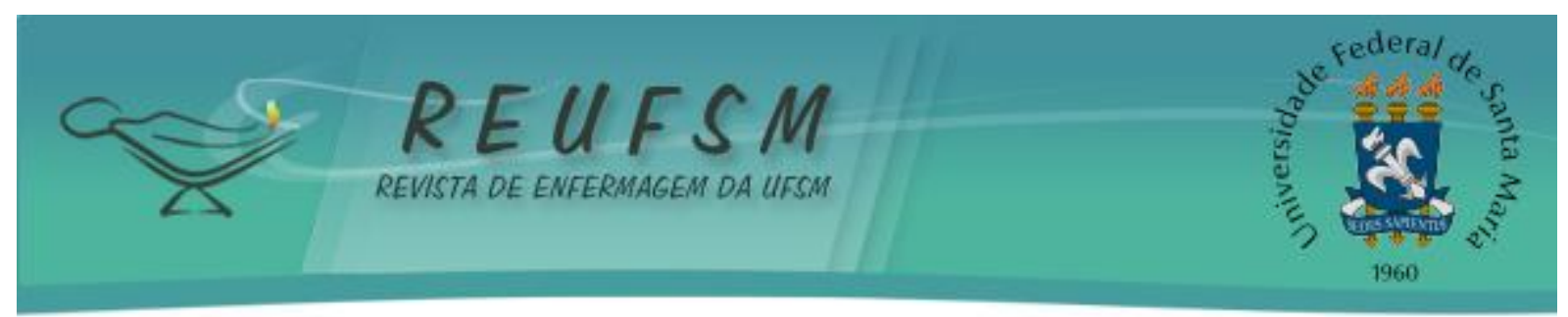

Ao mapear os riscos ergonômicos durante os procedimentos de movimentação e remoção de pacientes adultos nas unidades de internação investigadas, verificou-se que a $\mathrm{UCl}$ e a UTI são as que oferecem maior risco ergonômico aos trabalhadores, já que 76,9\% dos pacientes foram classificados com muito risco seguido por $15,4 \%$ com médio risco $(\mathrm{p}<0,05)$. Destaca-se a classificação de médio risco ergonômico para $50 \%$ dos pacientes observados nas unidades de Clínica Cirúrgica, Clínica Médica Il e Pronto Socorro.

No Pronto-Socorro $50 \%$ dos pacientes apresentavam médio risco ergonômico e $22,7 \%$ muito risco. Na Clínica Médica I, 76,9\% dos pacientes ofereciam pouco risco para a equipe de enfermagem. A maioria deles deambulava e fazia uso de equipamentos de infusão no momento de administração de quimioterápicos, o que permitia a livre movimentação e possibilitava maior autonomia ao paciente.

Observa-se que, em todas as unidades avaliadas, exceto a Clínica Médica I, o quantitativo de pacientes com médio e muito risco ergonômico é superior aos pacientes que oferecem pouco risco, o que significa maiores demandas para a equipe de enfermagem.

\section{DISCUSSÃO}

Em relação aos itens da escala, o peso foi a variável que obteve maior percentual para muito risco ergonômico para os trabalhadores de enfermagem $(46,3 \%)$ durante a movimentação e remoção dos pacientes. Nesse sentido, aspectos relacionados à demanda de trabalho da equipe de enfermagem, como o esforço físico, podem ocasionar importantes lesões na coluna vertebral. Estudo aponta que queixas de lombalgias estão relacionadas a problemas gerados pelo excesso de esforço físico, que podem ser agravados por postura inadequada do trabalhador. ${ }^{11}$

Pesquisa realizada para investigar a produção brasileira relacionada aos distúrbios musculoesqueléticos em trabalhadores de enfermagem evidenciou o aumento da prevalência de doenças osteomusculares em diversas ocupações, enfatizando as crescentes discussões acerca destas relacionadas ao trabalho. ${ }^{12}$ Os problemas de coluna podem ser agravados pela utilização de técnicas inadequadas (postura incorreta), a não utilização de materiais acessórios (prancha de transferência do paciente), que, associada à distância a ser percorrida, com o peso do paciente e com o número de vezes em que a tarefa se repete durante $o$ turno de trabalho, também podem influenciar na ocorrência de problemas de coluna.

Portanto, antes de iniciar atividades de movimentação e remoção de pacientes é importante avaliar e planejar de forma minuciosa todo o processo e, sempre que possível, usar recursos auxiliares que possibilitem ao trabalhador realizar suas atividades com segurança. Conforme o peso do paciente, serão adotadas determinadas posturas, avaliada a necessidade de materiais acessórios (plásticos acessórios, materiais antiderrapantes, pranchas para transferência) e dimensionado o quantitativo de trabalhadores. ${ }^{4}$

Em relação à altura dos pacientes, evidenciou-se médio risco ergonômico aos trabalhadores investigados. A estatura do paciente, muitas vezes, pode não ser considerada como relevante pelos trabalhadores da saúde no que se refere a movimentação e remoção de pacientes, entretanto, constitui-se em um fator que acentua o risco ergonômico. Ou seja, quanto mais alto for o paciente, maiores serão as exigências de força física por parte do trabalhador, o que exige um número maior de trabalhadores para realizar as atividades relacionadas ao manuseio desse paciente. ${ }^{9}$

O nível de consciência, mobilidade no leito, remoção e deambulação preservados reduzem o risco ergonômico, pois, quanto menor a dependência física do paciente, menor será o dispêndio de esforço físico por parte do trabalhador, exigindo apenas a supervisão dos trabalhadores de enfermagem, o que é considerado pouco risco ergonômico para 


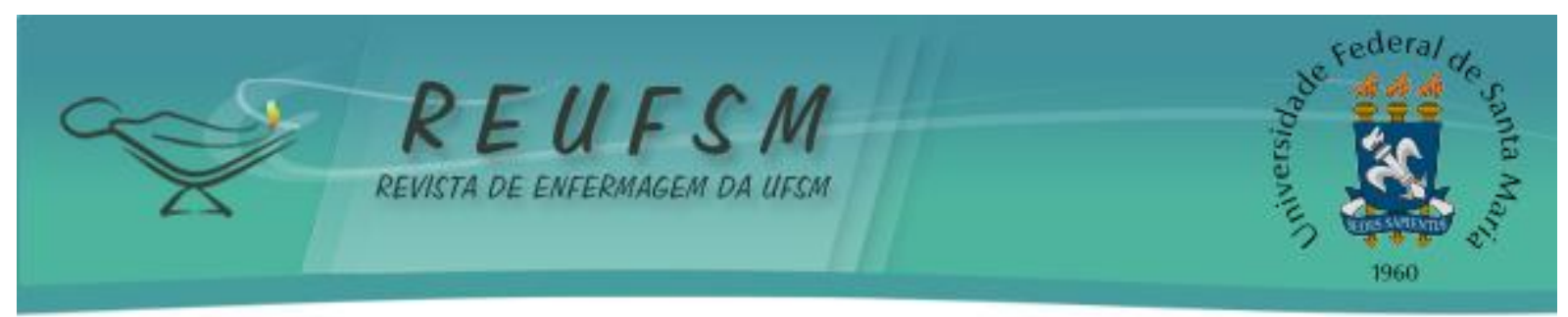

equipe de enfermagem. Neste estudo, foi observada variação de $9,8 \%$ a $41,5 \%$ de pacientes que necessitavam de algum auxílio ou eram dependentes da equipe de enfermagem para todas as atividades.

A tarefa de movimentação de pacientes é um fator de risco para lesões musculoesqueléticas entre os trabalhadores de enfermagem. A carência de materiais adequados para a realização desse procedimento associado ao número insuficiente de funcionários, falta de equipamento para elevação do paciente e posturas corporais incorretas são fatores que contribuem para os agravos a saúde dos trabalhadores de enfermagem.13

Quanto ao uso de acessórios e equipamentos, 60,2\% dos pacientes possuíam de dois a mais de cinco. É necessário considerar esses aspectos, pois eles dificultam a remoção dos pacientes, exigindo cuidado redobrado. Ainda, deve-se observar a utilização desses itens durante o processo de movimentação e remoção do paciente para que haja adequação ao meio de transporte. Isso requer do enfermeiro o planejamento das atividades junto aos demais membros da equipe de enfermagem, que deve sempre priorizar a segurança do paciente e a saúde do trabalhador.

Esse planejamento deve abordar a avaliação das condições do paciente, dos equipamentos, e o preparo do ambiente visando reduzir os riscos tanto para o paciente como para o trabalhador, evitando lesões e demais problemas de saúde nesses últimos.

Estudo sobre a intensidade da dor musculoesquelética e a capacidade para o trabalho realizado com trabalhadores de enfermagem sinaliza que no contexto do adoecimento dos trabalhadores de enfermagem, o distúrbio musculoesquelético é um dos fatores que contribuem para reduzir a capacidade para o trabalho. ${ }^{14}$ Dessa forma, os problemas relacionados a saúde do trabalhador devem ser identificados precocemente, para que possam ser implementadas medidas preventivas e interventivas, a fim de reduzir os danos para a saúde do trabalhador. ${ }^{15}$

Para realizar a avaliação ergonômica em uma instituição de saúde, precisam ser consideradas as especificidades de cada unidade e de seus pacientes. ${ }^{9}$ Os dados apresentados evidenciaram o dispêndio de esforço físico por parte dos trabalhadores da instituição pesquisada, uma vez que a soma das frequências de pacientes classificados com médio $(39,8 \%)$ e muito risco ergonômico $(17,1 \%)$ totalizou $56,9 \%$. Ressalta-se que há possibilidade de progressão ou regressão no quadro clínico do paciente quanto ao seu estado geral, o que possibilita maior ou menor risco ergonômico ao trabalhador.

Verificou-se que os pacientes internados na UCI e na UTI são os que oferecem maior risco ergonômico aos trabalhadores durante os procedimentos que exigem a movimentação e remoção desses pacientes. Estudo reforça que pacientes internados em UTI oferecem muito risco ergonômico para os trabalhadores de enfermagem, pois, na maioria das vezes, eles dependem totalmente da equipe de enfermagem. ${ }^{16}$ Esse fato pode ser explicado devido à complexidade do cuidado que os pacientes requerem, já que na maioria das vezes eles se encontram totalmente dependentes e sob sedação, em uso de ventilador mecânico e outros acessórios tais como, cateteres, sondas nasoentérica e vesical. Assim, a movimentação e remoção de pacientes em UTI são apontadas como fator contribuinte para adoecimento físico de trabalhadores de enfermagem. ${ }^{16}$

0 instrumento de avaliação de classificação de risco oferece subsídios para o planejamento dos procedimentos, auxilia o dimensionamento da equipe de enfermagem e faz atentar para a necessidade da utilização de equipamentos acessórios (elevadores, pranchas, entre outros).

Destaca-se ainda, neste estudo, a classificação de risco médio ergonômico para três unidades de internação. Na Clínica Cirúrgica, a maior unidade de internação adulto da instituição, o percentual elevado pode ser explicado pelo fato dos pacientes internados apresentarem restrições de movimentos devido às cirurgias, ao uso de catéteres com infusão 


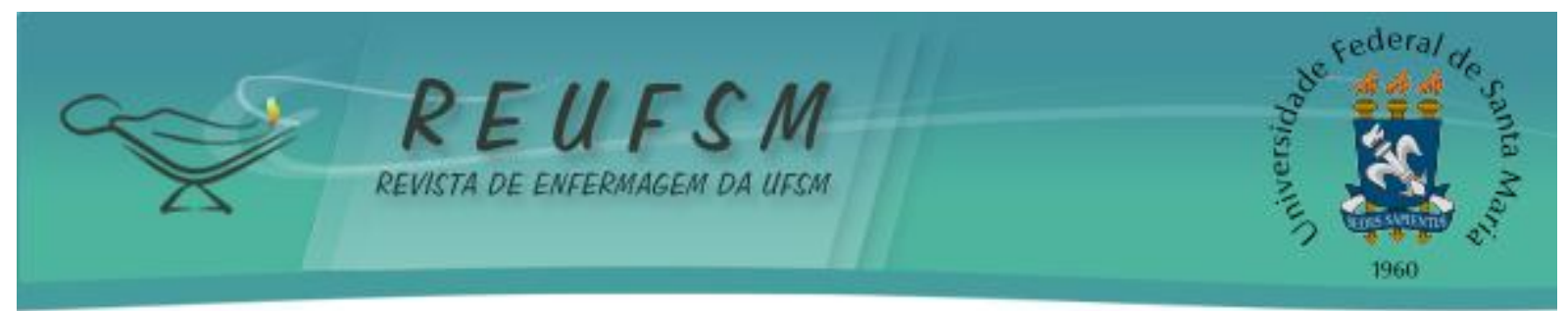

de soluções e/ou ao uso de drenos. Aqueles internados na Clínica Médica II apresentavam perdas funcionais devido às sequelas neurológicas, traumas medulares e doenças crônicas. Estas são condições que exigem dos trabalhadores de enfermagem planejamento dos cuidados e auxílio na movimentação e remoção dos pacientes. No Pronto-Socorro $50 \%$ dos pacientes apresentavam médio risco ergonômico e $22,7 \%$ muito risco.

Estudo realizado em uma unidade de pronto-socorro corrobora esses achados, ao identificar que $17,4 \%$ dos pacientes apresentavam nível de cuidado semi-intensivo e intensivo, ou seja, exigindo alta complexidade de cuidado. ${ }^{17}$

Observa-se que, em todas as unidades avaliadas, exceto a Clínica Médica I, o quantitativo de pacientes com médio e muito risco ergonômico é superior aos pacientes que oferecem pouco risco, o que significa maiores demandas para a equipe de enfermagem.

Evidencia-se que os trabalhadores que atuam no Pronto-Socorro, Clínica Cirúrgica, Clínica Médica II, UTI e UCI, necessitam participar de um rigoroso programa de capacitação ou treinamento antes de movimentar ou remover o paciente para que possam realizar esta atividade de maneira adequada de modo a promover segurança do paciente e sua própria segurança no ambiente de trabalho. Para este procedimento, o enfermeiro responsável pela equipe deve realizar supervisão direta e participativa.

Ressalta-se que, mesmo a unidade apresentando percentual maior de pacientes com pouco risco ergonômico, como na Clínica Médica I, ou com médio risco, como na Clínica Cirúrgica e Clínica Médica II, essas unidades também possuíam pacientes de muito risco ergonômico para a equipe, resultado semelhante ao encontrado em outro estudo. ${ }^{16}$

Mapear os riscos ergonômicos em unidades de internação permite identificar os setores com maior risco para os trabalhadores. A partir disso, gestores poderão planejar estratégias que favoreçam a saúde e a satisfação do trabalhador, a fim de melhorar a qualidade da assistência. Ressalta-se a importância da equipe de enfermagem identificar os problemas encontrados no ambiente de trabalho para propor soluções de modo que possam desempenhar suas tarefas com segurança, sem colocar sua saúde em risco em decorrência do trabalho. ${ }^{18}$

O enfermeiro, responsável pela gerência do cuidado e por toda a equipe de enfermagem, deve fazer um planejamento sistematizado da movimentação e remoção dos pacientes, o que poderá repercutir no rendimento e eficiência no trabalho, menor risco de adoecimento e maior qualidade de vida para os trabalhadores.

\section{CONCLUSÃO}

O estudo permitiu mapear os riscos ergonômicos para os trabalhadores de enfermagem nas diferentes unidades hospitalares, sendo que a Clínica Médica I apresentou maior percentual de pacientes com pouco risco ergonômico para os trabalhadores em comparação a Clínica Cirúrgica e Clínica Médica II que apresentavam médio risco. Além disso, pode-se identificar que o peso foi a variável que obteve maior percentual para muito risco ergonômico para os trabalhadores de enfermagem.

Nesse sentido, ações gerenciais, como adequação do mobiliário, capacitação dos trabalhadores, adequação da estrutura física, implementação de medidas de prevenção e utilização de materiais acessórios, além do dimensionamento de trabalhadores, podem minimizar esses riscos no ambiente hospitalar.

Portanto, o mapeamento dos riscos ergonômicos oferece subsídios tanto aos gestores quanto aos trabalhadores e ao Serviço de Segurança e Saúde do Trabalhador, na medida em que, ao se identificar esses riscos, podem ser desenvolvidos programas que objetivem a sua redução, por meio da sensibilização dos trabalhadores e gestores. 


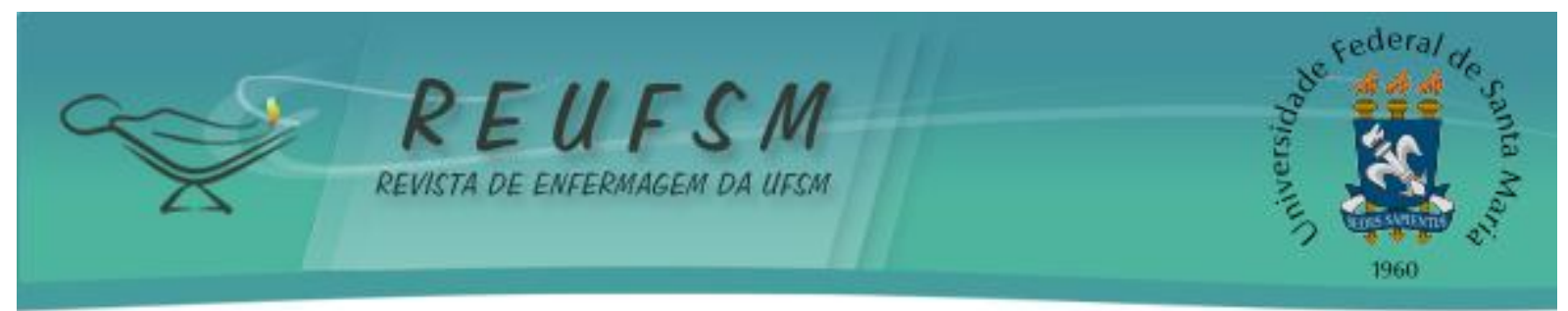

Por fim, este estudo permitiu a exploração inicial da temática que envolve a relação paciente-ambiente-trabalhador, na perspectiva de oferecer condições de trabalho que favoreçam a Saúde do Trabalhador e Segurança do Paciente.

\section{REFERÊNCIAS}

1. Mauro MYC, Paz AF, Mauro CCC, Pinheiro MAS, Silva VG. Condições de trabalho da enfermagem nas enfermarias de um hospital universitário. Esc Anna Nery. 2010 abr/jun;14(2):244-52.

2. Brasil. Ministério da Saúde. Portaria $n^{\circ} 1.823$, de 23 de agosto de 2012. Institui a Política Nacional de Saúde do Trabalhador e da Trabalhadora. Brasília; 2012.

3. Monteiro MS, Alexandre NMC. Work ability and low back pain among workers from a public health institution. Rev Gaúcha Enferm. 2009;30(2):297-302.

4. Silva LA, Secco IAO, Dalri RCMB, Araújo SA, Romano CC, Silveira SE. Enfermagem do trabalho e ergonomia: prevenção de agravos à saúde. Rev Enferm UERJ. 2011 abr/jun;19(2):317-23.

5. Brasil. Ministério do Trabalho. Secretaria de Inspeção do Trabalho. Portaria SIT n. ${ }^{\circ} 13$, de 21 de junho de 2007. Altera o Manual de aplicação da Norma Regulamentadora $n^{\circ} 17$. Brasília; 2007.

6. Fonseca NR, Fernandes RCP. Factores related to musculoskeletal disorders in nursing workers. Rev Latinoam Enferm [Internet]. 2010 nov/dec [acesso em 2013 jun 18];18(6):1076-83. Disponível em: http://www.scielo.br/pdf/rlae/v18n6/06.pdf.

7. Magnago TSBS, Lisboa MTL, Griep RH, Kirchhof ALC, Guido LA. Psychosocial aspects of work and musculoskeletal disorders in nursing workers. Rev Latinoam Enferm [Internet]. 2010 maio/jun [acesso em 2013 mar 15];18(3):429-35. Disponível em: http://www.scielo.br/pdf/rlae/v18n3/19.pdf.

8. Brasil. Ministério da Saúde. Conselho Nacional de Saúde. Resolução CNS n 196, de 10 de outubro de 1996. Aprova diretrizes e normas regulamentadoras de pesquisas envolvendo seres humanos. Brasília: Ministério da Saúde; 1996.

9. Radovanovic CAT, Alexandre NMC. Desenvolvimento de um instrumento para avaliar a movimentação e transferência de pacientes: um enfoque ergonômico. Rev Esc Enferm USP. 2002;36(3):231-9.

10. Radovanovic CAT, Alexandre NMC. Validação de um instrumento para avaliar a movimentação e transferência de pacientes. Cienc Cuid Saude. 2002;1(2):277-85.

11. Helfenstein Júnior M, Goldenfum MA, Siena C. Occupacional low back pain. Rev Assoc Med Bras [Internet]. 2010 [acesso em 2013 jun 20];56(5):583-9. Disponível: http://www.scielo.br/pdf/ramb/v56n5/en_v56n5a22.pdf.

12. Magnago TSBS, Lisboa MTL, Griep RH. Trabalho da enfermagem e distúrbio musculoesquelético: revisão das pesquisas sobre o tema. Esc Anna Nery Rev Enferm. 2008 set;12(3):560-5.

13. Lee SJ, Faucett J, Gillen M, Krause N. Musculoskeletal pain among critical-care nurses by availability and use of patient lifting equipment: an analysis of cross-sectional survey data. Int J Nurs Stud. 2013 dec;50(12):1648-57. 


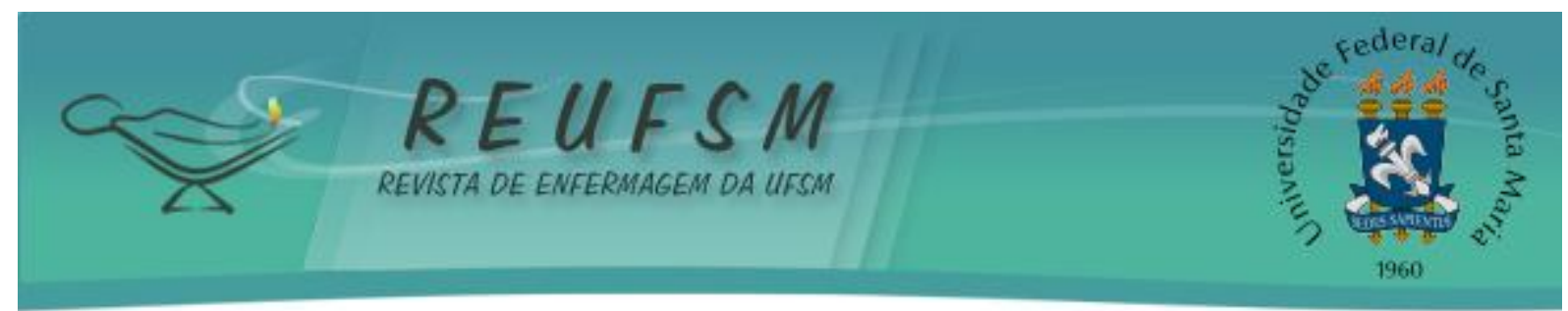

14. Magnago TSBS, Lima ACS, Prochnow A, Ceron MDS, Tavares JP, Urbanetto JS. Intensity of musculoskeletal pain and (in) ability to work in nursing. Rev Latinoam Enferm [Internet]. 2012 nov/dez [acesso em 2013 jun 23];20(6):1125-33. Disponível em: http: //www.scielo.br/pdf/rlae/v20n6/15.pdf.

15. Trindade LL, Lautert L. Syndrome of Burnout among the workers of the Strategy of Health of the Family. Rev Esc Enferm USP. 2010; 44(2):273-8.

16. Beserra FM, Souza AMA, Moreira DA, Alves MDS, D'Alencar BP. Significado do trabalho dos profissionais de enfermagem no hospital geral. Av Enferm. 2010 [acesso em 2013 jun 10];28(2):31-9. Disponível em: http://www.scielo.org.co/pdf/aven/v28n2/v28n2a03.pdf.

17. Zimmermann LP, Magnago TSBS, Urbanetto JS, Greco PBT, Viero NC, Vieira TG, et al. Avaliação do grau de dependência de cuidados de enfermagem dos pacientes internados em pronto-socorro. Rev Enferm UFSM [Internet]. 2011 [acesso em 2013 nov 16];1(2):15363. em: http://cascavel.ufsm.br/revistas/ojs-

2.2.2/index.php/reufsm/article/view/2449/1659.

18. Oliveira EB, Pinel JS, Gonçalves JBA, Diniz DB. Nursing work in hospital emergency units - psychosocial risks: a descriptive study. Online braz j nurs [Internet]. 2013;12(1):7388 [acesso em 2013 maio 27]. Disponível em: http://www.objnursing.uff.br/index.php/nursing/article/view/4046.

Data de recebimento: $30 / 07 / 2014$

Data de aceite: 04/02/2015

Contato do autor responsável: Tânia Solange Bosi de Souza Magnago Endereço postal: Centro de Ciências da Saúde/CCS Avenida Roraima, 1000 Prédio 26, Sala 1339, Cidade Universitária Bairro Camobi, CEP: 97105-9000, Santa Maria (RS), Brasil E-mail: tmagnago@terra.com.br 